\title{
Estudio de la comunidad de oribátidos (Acari: Oribatida) en dos parches de tala (Celtis ehrenbergiana) del sudeste bonaerense
}

\author{
Natalia A. Fredes \\ Departamento de Biología, Universidad Nacional de Mar del Plata; CONICET.
}

\begin{abstract}
RESUmen. Los talares son bosques xéricos localizados a lo largo de la costa bonaerense argentina. Desde hace más de 400 años han estado sometidos a una degradación fuerte, lo cual generó un ambiente fragmentado, con parches de bosque de diferente superficie embebidos en una matriz de pastizal. Los objetivos del presente trabajo fueron: a) ampliar el conocimiento de la fauna de oribátidos de los talares, y b) analizar el efecto de la fragmentación del hábitat sobre la diversidad, la riqueza, la abundancia y la estructura de la comunidad de oribátidos en dos parches de tala del sudeste bonaerense. Se analizó la comunidad de oribátidos de dos parches cercanos, de diferente área (grande: $\mathrm{Gr}$, chico: $\mathrm{Ch}$ ) y la matriz de pastizal circundante (Ma) en la reserva Municipal de Laguna de los Padres, Mar del Plata. Se identificaron 51 especies de oribátidos, con valores de riqueza similares en los tres ambientes. Oppiidae y Scheloribatidae fueron las familias con más especies. La diversidad $\left(\mathrm{H}^{\prime}\right)$ fue mayor en los parches de tala $(\mathrm{Gr}=1.58 ; \mathrm{Ch}=1.59)$ que en la matriz $(\mathrm{Ma}=0.91)$. La densidad de oribátidos fue mayor en $\mathrm{Gr}$ (36500 individuos $/ \mathrm{m}^{2}$ ); en Ch y en Ma, los valores medios fueron 50\% y 75\% menores, respectivamente. La diferencia global en densidad es consecuencia de la disminución del tamaño poblacional de la mayoría de las especies. Estacionalmente, la comunidad de oribátidos de Gr contó con más especies "ubicuas" (20 especies) que las de Ch y Ma (10 especies). Las comunidades de oribátidos estudiadas estuvieron condicionadas principalmente por el tipo de ambiente (pastizal o bosque), con un patrón de distribución de las especies muy similar, pero con variaciones estacionales más marcadas en el pastizal. En los parches de bosque, el área no afectó la distribución ni la diversidad de especies, pero sí la densidad de especies, que fueron más pequeñas en el parche menor. La evaluación de la fragilidad de estas poblaciones podría ser un punto de inicio a la hora de emprender programas de conservación.
\end{abstract}

[Palabras clave: ácaros, comunidad edáfica, talares, Argentina]

Abstract. Study of the oribatid mite (Acari: Oribatida) community from two patches of tala (Celtis ehrenbergiana) from the southern of Buenos Aires. "Talares" are xeric forests located along the coast of Buenos Aires province, Argentina. Since $>400$ years ago, they have been subject of a severe degradation, resulting in a fragmentary landscape of forest patches surrounded by a grassland matrix. The aims of this study were: a) to enhance the knowledge of oribatid mites in the talares, and b) to analyze the effect of habitat fragmentation on diversity, richness, abundance and structure of the oribatid mite community. I analyzed oribatids in three sites: two different-size close forest patches (big: $\mathrm{Gr}$, small: $\mathrm{Ch}$ ) and the surrounded grassland matrix (Ma) in Laguna de los Padres reserve, Mar del Plata. A total of 51 species of oribatids were identified, with similar values of richness in all sites. Oppiidae y Scheloribatidae were the families with more species. Diversity $\left(\mathrm{H}^{\prime}\right)$ was greater in the forest patches $(\mathrm{Gr}=1.58 ; \mathrm{Ch}=1.59)$ than in the matrix $(\mathrm{Ma}=0.91)$. Density of oribatid mites was higher in $\mathrm{Gr}\left(36500\right.$ individuals $\left./ \mathrm{m}^{2}\right)$; in $\mathrm{Ch}$ and $\mathrm{Ma}$, densities were $50 \%$ and $75 \%$ lower. This global difference is a consequence of reduced population sizes in most species. According to seasonal variations, in Gr there were more "ubiquitous" species (20) than in Ch y Ma (10). Summarizing, the community of oribatid mites were primarily conditioned by the type of environment (grassland vs. forest), with similar distribution patterns of species but with a marked seasonality in the grassland matrix. Among forest patches, area did affect oribatid mites density through a reduction in most population sizes. The evaluation of the fragility of these populations could be a starting point to undertake conservation programs.

[Keywords: mites, edaphic community, talares, Argentina]

Editor asociado: Fernando Milesi

$\bowtie$ nfredes@mdp.edu.ar
Recibido: 7 de noviembre de 2015

Aceptado: 18 de abril de 2016 


\section{INTRODUCCIÓN}

Los ácaros oribátidos son los microartrópodos más abundantes en los suelos de bosque. Representan la fracción de la mesofauna con un ancho corporal entre 0.1 y $2 \mathrm{~mm}$ (Swiff et al. 1979; Coleman et al. 2004). Su abundancia en el suelo alcanza los 150000 individuos $/ \mathrm{m}^{2}$ en bosques templados, con una diversidad superior a 100 géneros en 100 $\mathrm{g}$ de suelo (Coleman et al. 2004). En bosques templados, la densidad de oribátidos varía de forma estacional debido principalmente a la humedad del suelo (Badejo 1990), a la cantidad de materia orgánica (Andres and Athias-Binche 1998; Hasegawa 2001) y a la disponibilidad de alimento (Block 1966; AlAssiuty et al. 1993). Su importancia en sistemas naturales radica en su destacada participación en la fragmentación y descomposición de la materia orgánica, en la regulación de la comunidad de bacterias y hongos, y en el transporte de esporas de hongos (Petersen and Luxton 1982; Lussenhop 1992). Por su abundancia y rol en los procesos edáficos, los cambios en la estructura de la comunidad de los oribátidos han sido usados para evaluar el grado de disturbio de un ambiente y sus posibles causas (Behan-Pelletier 1999; Gergócs and Hufnagel 2009).

La fragmentación del hábitat es un proceso a nivel de paisaje, fuertemente asociado a la pérdida y / o a la disminución de la riqueza de especies, a la pérdida de diversidad biológica y al deterioro global de la calidad del hábitat en relación con el tamaño y el estado de los parches remanentes (Sauders et al. 1991; Debinski and Holt 2000). Las comunidades edáficas, y en particular la comunidad de ácaros, se han utilizado como un modelo de estudio experimental tendiente a evaluar los efectos de la fragmentación del hábitat sobre su diversidad, riqueza y abundancia (Gonzales et al. 1998; Gonzales and Chaneton 2002; Hoyle and Harborne 2005). Debido a la complejidad de un ambiente natural, son pocos los estudios que evalúan los efectos de la fragmentación a escala de paisaje. Dentro de ellos, algunos se focalizan en algún aspecto de la fragmentación, como la colonización post-disturbio (Zaitsev et al. 2014), la relación entre calidad nutricional en los parches y las comunidades de oribátidos existente, o las diferencias en composición de especies bajo parches de bosque y pastizal (Ferreira et al. 2012).

Los talares son bosques xéricos que se localizan a lo largo de la costa bonaerense desde el Delta del Paraná hasta las inmediaciones de la ciudad de Mar del Plata, Argentina. Desde hace más de 400 años, estos talares han estado sometidos a una fuerte degradación debido a la tala indiscriminada, a la extracción de conchilla en los talares del norte de la provincia de Buenos Aires, al clareado para la utilización del suelo con fines agrícola-ganaderos y a la introducción de especies arbóreas exóticas (Parodi 1940b; Vervoorst 1967; Stupino et al. 2004; Torres Robles and Arturi 2009). Este proceso de degradación ha producido una fuerte reducción de su extensión original (Parodi 1940a, 1940b), configurando el actual paisaje fragmentado. Al presente, en la provincia de Buenos Aires, muchos de los fragmentos originales de los talares están protegidos bajo la categoría de reserva municipal, provincial o nacional. Sin embargo, pese a estas acciones en favor de la conservación, se desconoce la situación de estos bosques (Torres Robles and Tur 2006), con el consiguiente impedimento de realizar programas para su manejo y/o conservación.

Por su biogeografía, los talares bonaerenses son considerados una región extrazonal dentro de la región Pampeana, aunque comparte muchos elementos florísticos con la región del Espinal (Cabrera 1971); entre ellos, su especie emblemática: el tala o Celtis ehrenbergiana (Klotzsch) (=Celtis tala). Haene (2006), al estudiar las especies florísticas de los talares bonaerenses, dividió el sistema en dos subunidades: talares de barranca y talares de lomada. Los primeros se extienden desde el Delta del Paraná hasta la ciudad de La Plata, y los de lomada desde La Plata hasta la ciudad de Mar del Plata. Los de barranca, también denominados algarrobales, se asientan sobre la barranca que separa la región pampeana y el cauce de los ríos Paraná, Paraná de las Palmas y de la Plata y se extienden en dirección sur ocupando las terrazas bajas lindera al río de La Plata. Son los de mayor diversidad biológica (Haene 2006) y donde se registran la mayor proporción de parches mixtos de $C$. ehrenbergiana junto a elementos típicos de la región del Espinal. En los talares de lomada, los parches de C. ehrenbergiana son generalmente monoespecíficos y se encuentran rodeando cuerpos de agua (Parodi 1940a; Torres Robles and Tur 2005). Los talares de barranca se desarrollan sobre bancos de conchillas y depósitos marinos, y los de lomada sobre dunas maduras de limo (Haene 2006). 
Latitudinalmente, una de las últimas expresiones de los talares de lomada se localiza en la reserva Municipal de la Laguna de los Padres, a $14 \mathrm{~km}$ de la ciudad de Mar del Plata (Pdo. General Pueyrredon, Buenos Aires). Allí se reconocen dos parches monoespecíficos: uno de mayor área que rodea el extremo este de la laguna y otro, de menor área, alejado $300 \mathrm{~m}$ de la laguna. Estos dos parches están embebidos en una matriz de pastizal con algunos sectores con predominio de elementos exóticos como ligustros y/o acacias.

Considerando que los oribátidos representan el taxón más abundante en el suelo, con una participación activa en los procesos de descomposición, el estudio de la estructura comunitaria en un ambiente fragmentado, como es el caso de los talares, puede brindar elementos que permitan tanto ver el estado actual de los talares como implementar prácticas de manejo y/o conservación. Los objetivos del presente trabajo fueron: a) ampliar el conocimiento de la fauna de los talares a través de la descripción de la comunidad edáfica de oribátidos de los talares de lomada del sudeste bonaerense, y b) analizar el efecto de la fragmentación del hábitat sobre la diversidad, la riqueza, la abundancia y la estructura de la comunidad de oribátidos en dos parches de tala de Laguna de Los Padres. Las hipótesis propuestas son: a) los talares albergan una comunidad de oribátidos más diversa y abundante que en la matriz circundante, b) la fragmentación del hábitat afecta negativamente a la comunidad de oribátidos, reduciendo su diversidad, abundancia y riqueza en relación directa con la disminución del área continua de bosque. Por lo tanto, se espera que las comunidades de oribátidos de los parches de bosque sean estructuralmente más similares entre sí que con el pastizal circundante y que el parche de mayor área tenga valores de riqueza, diversidad y/o abundancia mayores que los del parche menor.

\section{MATERIALES y MÉTODOS}

\section{Sitio de estudio}

Las muestras fueron colectadas en las inmediaciones de la Laguna de los Padres (375' S; 57²4'31" O), Mar del Plata, Buenos Aires, Argentina. Se consideraron los dos parches monoespecíficos de C. ehrenbergiana que existen en el área: uno de $3000 \mathrm{~m}^{2}$ (Gr) y otro de $1500 \mathrm{~m}^{2}$ (Ch). Ambos parches se hallan en las inmediaciones de la Laguna de los Padres: el parche mayor rodea por el oeste la laguna y el menor dista de éste $\sim 200$ $\mathrm{m}$. También se realizó un muestreo en un sector al azar en el área de pastizal (Ma) entre los parches. Para analizar el estado sucesional de los parches se realizó un relevamiento de ejemplares de $C$. ehrenbergiana y se midió el diámetro a la altura del pecho (DAP) de $30(\mathrm{Gr})$ y $18(\mathrm{Ch})$ individuos. La edad de cada parche se estimó considerando la mayor frecuencia relativa de clases diamétricas. Para el cálculo de la edad se consideró la estimación de crecimiento en diámetro $(0.5 \mathrm{~cm} / \mathrm{año})$ dada por Arturi and Goya (2004).

\section{Extracción e identificación de oribátidos}

Se realizaron ocho muestreos con una frecuencia trimestral desde julio de 2008 hasta mayo de 2010. Previo a la toma de muestra se calculó la cantidad de muestras representativas de acuerdo al área de cada parche (Borcard 1988). Para el caso de la matriz se consideró la cantidad media de muestras de cada parche. En cada muestreo se extrajeron un total de 18 muestras repartidas equitativamente en centro y periferia del parche $(\mathrm{Gr}=8 ; \mathrm{Ch}=4 ; \mathrm{Ma}=6)$, colectándose un total de 144 muestras. Cada muestra consistió en un cilindro de suelo de $6 \mathrm{~cm}$ de diámetro por $5 \mathrm{~cm}$ de profundidad. Las muestras fueron tomadas con pala, trasladadas al laboratorio en envases plástico y colocadas en embudos Berlese durante 12 días, a temperatura ambiente y sin régimen de luz. La determinación de los oribátidos se realizó con un microscopio óptico Olympus CX30, mediante el montado y aclarado de los especímenes en ácido láctico (1:1). La identificación de las especies se realizó con las claves de Balogh y Balogh (1988, 1992, 1990), Subías y Balogh (1989) y Niedbała (2004). La identificación se corroboró mediante la comparación con las descripciones originales y/o redescripciones de cada especie. Los especímenes analizados se hallan en la colección particular de la autora.

\section{Análisis estadísticos}

Para cada ambiente se calcularon riqueza absoluta (S) y estimada (Chao 1, Jackknife y Bootstrap), y los índices de diversidad de Shannon-Winner ( $\left.\mathrm{H}^{\prime}\right)$ y paridad de Pielou (J') (Magurran 2004). Las hipótesis estadísticas sobre las diferencias en densidad, riqueza y diversidad se pusieron a prueba mediante la prueba no paramétrica de Kruskal-Wallis. 
La distribución temporal de las especies se analizó considerando cuatro categorías: ubicuas (si se registraron durante todo el año), frecuentes (si se registraron en tres estaciones), poco frecuentes (si se registraron en dos) y raras (si se registraron en solo una estación). Se realizaron gráficos rango de abundancia (RAD por sus siglas en inglés rank-abundance distribution) considerando la abundancia relativa de aparición de las especies en las muestras de cada ambiente (Gr, Ch y Ma).

Para cuantificar la similitud entre los ambientes en función de las especies compartidas se calculó el índice de diversidad $\beta$ de Simpson. Este índice, de acuerdo a Koleff et al. (2003), prioriza la cantidad de especies compartidas (a) más que las exclusivas de cada ambiente (b y c). Su fórmula es:

$$
\beta=\frac{\min (b, c)}{\min (b, c)+a}
$$

donde ' $a$ ' es la cantidad de especies compartida por los ambientes, 'b' es el número de especies halladas únicamente en el primer ambiente $y^{\prime}$ c $c$ es el número de especies halladas únicamente en el segundo ambiente. El índice toma valores desde 0 , cuando los ambientes no tienen especies exclusivas y por lo tanto no hay recambio de especies entre ambientes (la diversidad $\beta$ es nula) hasta 1 , cuando no hay especies compartidas $(a=0)$ y la diversidad $\beta$ es máxima.

La estructura de la comunidad se analizó mediante un análisis de correspondencias (CA) considerando la abundancia de las especies en cada uno de los tres ambientes y a través de las estaciones. Los análisis estadísticos se llevaron a cabo con el software R (www.r-project.org) y el paquete vegan (Oksanen et al. 2011).

\section{REsultados}

\section{Medidas diamétricas y edad de los parches}

En Gr predominaron ejemplares de $C$. erenbergiana de 10-12 cm de diámetro, hallándose un ejemplar con un diámetro de $37 \mathrm{~cm}$. En Ch predominaron los ejemplares de 6-7 $\mathrm{cm}$ de diámetro con un máximo de 22 $\mathrm{cm}$. La edad estimada de los parches fue 20-24 años para Gr y 12-14 para Ch.

\section{Comunidad de oribátidos}

Se identificaron un total de 51 especies distribuidas en 44 géneros y 30 familias (ver Anexo). Oppiidae y Scheloribatidae fueron las familias más relevantes en cuanto a número de especies. En Gr, Physobates spinipes, Tectocepheus minor, Scheloribates elegans y Scheloribates praeincisus acuticlava fueron las especies más abundantes, representando $75 \%$ de la abundancia total. En Ch el mismo porcentaje lo acumularon cinco especies: P. spinipes, Ramusella (I.) merimna, Eremulus crispus, Oxyoppia (O.) suramericana y S. praeincisus acuticlava. En Ma Hemileius suramericanus, $S$. praeincisus acuticlava, $P$. spinipes, O. (O.) suramericana y Oppiella nova, representaron el mismo porcentaje de abundancia total. La cantidad promedio de especies por muestra fue 10,9 y 4 para $G, C h$ y Ma respectivamente. Los gráficos RAD por ambiente mostraron patrones muy similares de distribución de especies (Figura 1): 2-4 especies con abundancias relativas superiores al 10\%, y 7-13 especies con una abundancia de $1-10 \%$. La única diferencia a remarcar fue la presencia en Gr de especies con abundancias relativas inferiores al $0.05 \%$, ausentes en $\mathrm{Ch}$ y Ma.

\section{Densidad y diversidad de oribátidos}

En los parches de bosque la densidad media de oribátidos fue 36500 individuos $/ \mathrm{m}^{2}$ en Gr y 18600 individuos $/ \mathrm{m}^{2}$ en $\mathrm{Ch}$, con registros máximos en primavera (115000 individuos/ $\mathrm{m}^{2}$ ) en Gr y en verano (61000 individuos $/ \mathrm{m}^{2}$ ) en Ch (Figura 2a y 2b). En la matriz la densidad media fue 12600 individuos $/ \mathrm{m}^{2}$, con valores máximos en otoño (28300 individuos $/ \mathrm{m}^{2}$ ) (Figura 2c). La riqueza absoluta fue muy similar en los tres ambientes, levemente mayor en Gr. En los tres ambientes, los estimadores de riqueza Jackknife y Bootstrap cuantificaron los menores desvíos. La diversidad fue mayor en los parches de bosques respecto de la matriz $\left(\mathrm{X}^{2}=48.7, \mathrm{P}<0.001\right)$. El índice de paridad de especies $\left(J^{\prime}\right)$ no fue diferente entre los ambientes (Tabla 1).

\section{Estacionalidad}

Del total de especies halladas en Gr, 47\% estuvieron presentes en todas las estaciones (ubicuas), mientras que en $\mathrm{Ch}$ y en Ma las especies ubicuas no superaron el 30\% (Figura 3). Dentro de las especies ubicuas, cuatro (P. spinipes, T. velatus, $R$. (I.) merimna y $S$. praeincisus acuticlava) se hallaron en los tres ambientes. En los tres ambientes las estaciones 


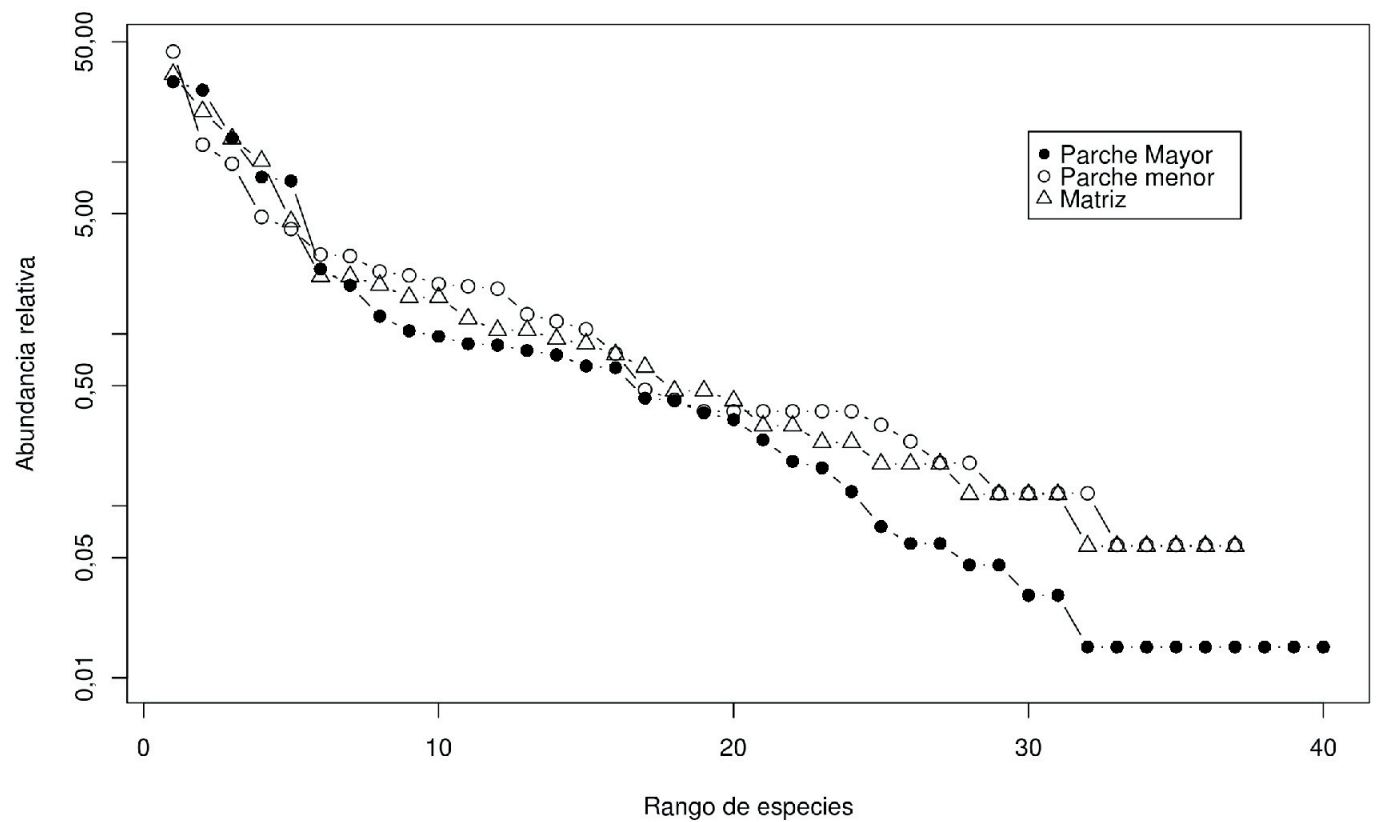

Figura 1. Rango de abundancias de especies (RAD) por ambiente. La abundancia relativa (\%) se expresa sobre una escala logarítmica.

Figure 1. Rank-abundance distribution of species (RAD) in each environment. Relative abundance (\%) is expressed on a logarithmic scale.
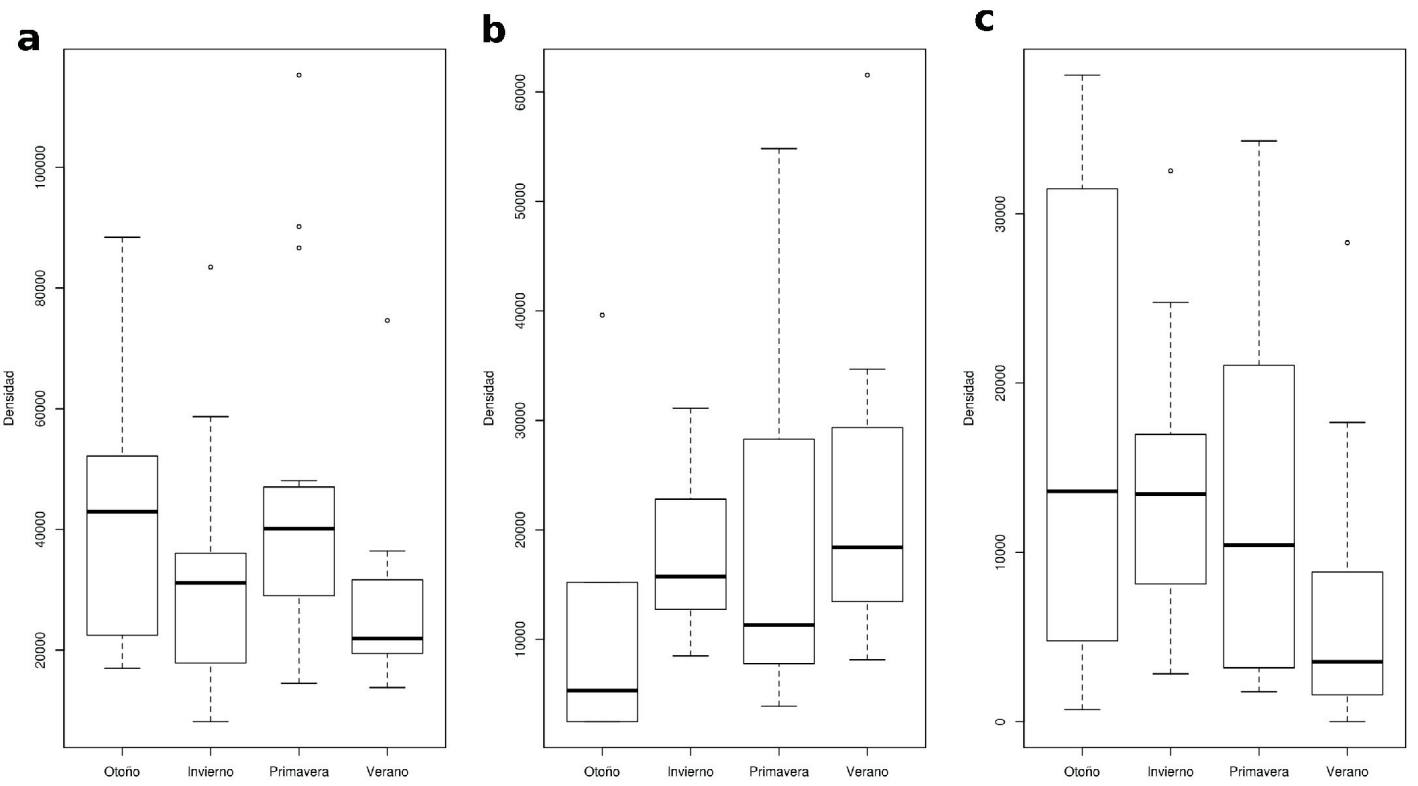

Figura 2. Densidad (individuos $/ \mathrm{m}^{2}$ ) de oribátidos en cada estación: a) parche mayor de bosque de tala (Gr); b) parche menor (Ch); c) matriz de pastizal (Ma).

Figure 2. Density of oribatid mites (individuals $\left./ \mathrm{m}^{2}\right)$ by season: a) big tala forest patch $(\mathrm{Gr})$; b) small patch $(\mathrm{Ch})$; $\left.\mathrm{c}\right)$ grassland matrix (Ma). 
Tabla 1. Riqueza, diversidad y paridad en el parche mayor $(\mathrm{Gr})$, el parche menor $(\mathrm{Ch})$ y la matriz (Ma). Letras distintas significan diferencias significativas de acuerdo a la prueba de Kruskal-

\begin{tabular}{|c|c|c|c|c|c|c|}
\hline & Absoluta & Chao 1 & Jackknife & Bootstrap & $\mathrm{H}^{\prime}$ & $\mathrm{J}^{\prime}$ \\
\hline $\mathrm{Gr}$ & 40 & $76 \pm 33.4$ & $52 \pm 3.41$ & $45 \pm 1.75$ & $1.58^{\mathrm{a}}$ & $0.68^{\mathrm{a}}$ \\
\hline $\mathrm{Ch}$ & 37 & $97 \pm 71.1$ & $47 \pm 3.77$ & $41 \pm 1.85$ & $1.59^{\mathrm{a}}$ & $0.74^{\mathrm{a}}$ \\
\hline Ma & 37 & $44 \pm 6$ & $48 \pm 4.05$ & $42 \pm 2.77$ & $0.91^{\mathrm{b}}$ & $0.64^{\mathrm{a}}$ \\
\hline
\end{tabular}
Wallis $(\alpha=0.05)$.

Table 1. Richness, diversity and evenness in the big forest patch $(\mathrm{Gr})$, the small patch $(\mathrm{Ch})$ and the grassland matrix (Ma). Different letters mean significant differences according to Kruskal-Wallis' test $(\alpha=0.05)$.

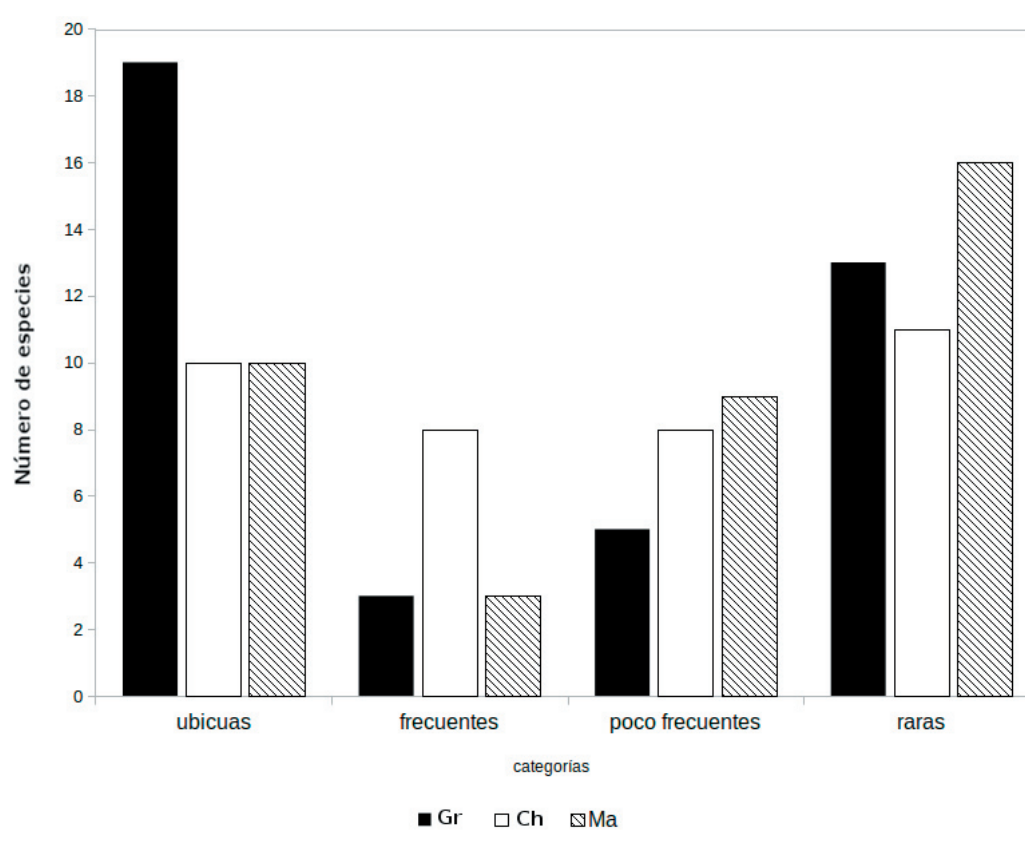

Figura 3. Riqueza (número de especies) de oribátidos de acuerdo a su frecuencia de detección en los muestreos estacionales en cada ambiente. $\mathrm{Gr}=$ parche de bosque mayor; $\mathrm{Ch}=$ parche menor; $\mathrm{Ma}=$ matriz (pastizal). Ubicuas=estuvieron presentes todo el año; frecuentes=en tres estaciones; poco frecuentes =en dos; raras=en una sola estación.

Figure 3. Richness (number of species) of oribatid mites species according to their seasonal frequency in each environment. $\mathrm{Gr}=$ big forest patch; $\mathrm{Ch}=$ small patch;Ma $=$ grassland matrix. Ubiquitous (ubicuas) $=$ species found in all seasonal samples; frequent (frecuentes) $=$ species present in three seasons; less frequent (poco frecuentes $)=$ species present in two seasons; rare (raras)=species present in only one season.

de invierno y otoño registraron 4-6 especies más, siendo Ch el ambiente que presentó mayor cantidad (7 especies más en invierno respecto a primavera y verano).

En cuanto a la diversidad por estación, en Gr en otoño se registró la máxima diversidad $\left(\mathrm{H}^{\prime}=1.7\right)$ y en verano la mínima $\left(\mathrm{H}^{\prime}=1.5\right)$. Para $\mathrm{Ch}$ la diversidad máxima y mínima correspondió a invierno $\left(\mathrm{H}^{\prime}=1.8\right)$ y otoño $\left(H^{\prime}=1.3\right)$ respectivamente. Finalmente, en la matriz el máximo valor se registró en primavera $\left(\mathrm{H}^{\prime}=1.1\right)$ y el mínimo en otoño y verano $\left(H^{\prime}=0.8\right)$. Los valores de paridad estacionales no registraron alteraciones de relevancia.

\section{Estructura de la comunidad}

De las 51 especies identificadas, 26 estuvieron en los tres ambientes y solo 3 fueron exclusivas de los talares. Los valores del índice de diversidad $\beta$ de Simpson nunca superaron 0.22 (Tabla 2), lo que refleja un bajo recambio de especies entre los ambientes.
El análisis de correspondencias (CA) acumuló en sus primeros tres ejes el $69 \%$ de la variabilidad total. Las muestras se separaron por ambiente $(\mathrm{Gr}, \mathrm{Ch}$ y $\mathrm{Ma})$ y a cada agrupación se asoció un ensamble de especies. En los dos primeros ejes (53\%) se separaron la matriz de los parches de bosque y en el segundo eje se segregaron los parches entre sí (Figura 4). En el tercer eje, las muestras de Ma se separaron en dos grupos: uno con las muestras de invierno y verano y otro con las de otoño y primavera. Las especies más vinculadas a Gr fueron Nothrus anauniensis, $N$. becki, S. elengans, Graptoppia (G.) alzueti, Lamellobates aff. quadricornis, Urubambates paraguayensis y T. minor. A Ch se vincularon lasespecies Galumnaaff.circularis, Protoribates capuccinus,Teratoppiabrevipectinata,Fosseremus laciniatusy Eremuluscrispus;mientrasquea Ma se vincularon Hemileius suramericanus, O. (O.) suramericana, O.nova, Anderemaeuschilensis, Mancoribates rostropilosus, S. praeincisus acuticlava y Oribatula translineata. 

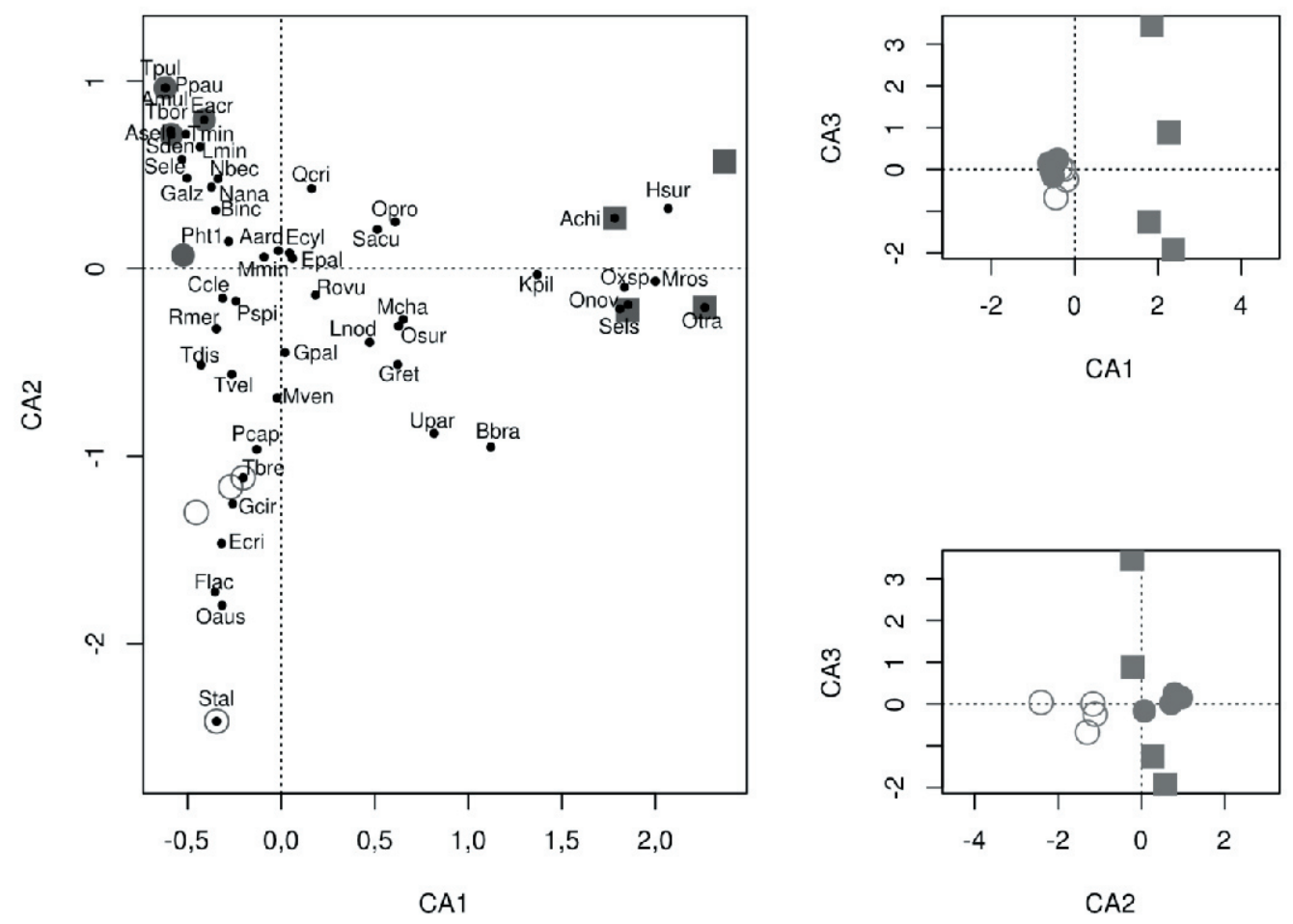

Figura 4. Gráficos del análisis de correspondencias (CA) considerando los tres primeros ejes. Los círculos llenos representan las muestras estacionales del parche mayor (Gr), los círculos las estaciones del parche menor (Ch) y los cuadrados llenos las de la matriz (Ma). Ver anexo 1 para los nombres de las especies.

Figure 4. Biplots of correspondence analysis (CA) considering the first three axes. Fill circles represent the samples of big patch (Gr), empty circles for samples in the small patch $(\mathrm{Ch})$ and filled squares for the samples of grassland matrix (Ma). See annex 1 for species' full names.

Tabla 2. Valores de diversidad beta de Simpson por pares de ambientes. Parche mayor (Gr), el parche menor (Ch) y la matriz (Ma).

Table 2. Simpson beta diversity values by pairs of sites. Big forest patch $(\mathrm{Gr})$, small patch $(\mathrm{Ch})$ and grassland matrix (Ma).

\begin{tabular}{lll}
\hline & Gr & Ch \\
\hline Gr & 0 & \\
Ch & 0.216 & 0 \\
$\mathrm{Ma}$ & 0.189 & 0.162 \\
\hline
\end{tabular}

\section{DISCUSIÓN}

\section{Diversidad, densidad y estructura comunitaria}

La comunidad de oribátidos de los talares de Laguna de los Padres albergó una alta riqueza de especies, cuyas estimaciones - muy cercanas a los valores absolutos obtenidospermiten aseverar que el esfuerzo de muestreo ha sido adecuado para relevar casi totalidad de las especies existentes en los ambientes estudiados (Tabla 1). A nivel de paisaje la diversidad $\beta$ fue baja, con aproximadamente la mitad de las especies presentes en los dos parches y en la matriz de pastizal (Tabla 2). Pese a que el talar y el pastizal presentaron un pool de especies comunes y valores de riqueza comparables, en los parches de tala la diversidad $\left(\mathrm{H}^{\prime}\right)$ y densidad de individuos fueron superiores a los registrados para la matriz, confirmando la hipótesis propuesta.

La densidad de oribátidos fue más alta en el parche mayor $(\mathrm{Gr})$, y se registró una reducción de $50 \%$ en el parche menor y cerca de $75 \%$ en la matriz de pastizal. Las diferencias en densidad entre la matriz y los parches son comparables a los reportados por Ferreira et al. (2012) para la comunidad de oribátidos en bosques fragmentados de Brasil, en comparación con la matriz de sabana amazónica cercana. En cuanto a la diferencia entre los parches, por un lado, contrasta con el obtenido experimentalmente por Hoyle and Harborne (2005), mientras que concuerda con lo hallado por Koteja et al. (1994). Estos últimos autores atribuyen las diferencias en densidad entre parches de distinto tamaño explícitamente a la reducción de la capacidad de carga del ambiente, y vinculado estrictamente a la cantidad de recurso alimentario y disponibilidad de micrositios para la oviposición requeridos para el sustento de la comunidad de oribátidos. 
En contraposición a la predicción hecha, la menor densidad de individuos en el parche menor no se reflejó en una disminución en la riqueza de especies, en la diversidad o en la paridad (Tabla 1). Sin embargo, un interesante hallazgo surge cuando se analiza en detalle la densidad de cada una de las especies (Figura 5): la reducción en densidad global de individuos es consecuencia de una reducción en el tamaño de la mayoría de las poblaciones, es decir, que en el parche con menor área las poblaciones contaron con menos individuos. Por ejemplo, $P$. spinipes - especie dominante en ambos parches - redujo su densidad poblacional $25 \%$ en el parche menor respecto del mayor, S. praeincisus acuticlava, con una población superior a 1500 individuos $/ \mathrm{m}^{2}$ en el parche mayor, redujo su número a 400 individuos $/ \mathrm{m}^{2}$ en el parche menor, y Tectocepheus minor, cuya población en Gr fue 5000 individuos $/ \mathrm{m}^{2}$, en el parche menor no superó los 300 individuos / $\mathrm{m}^{2}$. En el contexto de la conservación de los talares bonaerenses y la necesidad de implementar programas para su conservación y/o manejo, esta disminución generalizada de la densidad poblacional podría implicar una pérdida de diversidad filogenética que podría comprometer la subsistencia de la comunidad a largo plazo.

Respecto de la dominancia y distribución de las especies en la matriz y los parches, los valores de equitatividad ( $\left.J^{\prime}\right)$ fueron muy similares y sólo se observaron diferencias en aquellas especies con abundancias relativas inferiores al $0.05 \%$, que estuvieron ausentes en el pastizal y en el parche menor (Figura 1). Estos resultados pueden estar indicando que las diferencias temporales (i.e., la diferencia en edad de los parches aquí registrados) entre los parches no están condicionando la dominancia y distribución de las especies en Ch. Es decir, se puede considerar que existe una misma probabilidad de colonización de nuevas especies en uno u otro parche, y que, por lo tanto, la ausencia de las especies con abundancias inferiores a $0.05 \%$ en el parche menor no es debido a la edad del parche sino probablemente a limitaciones en la capacidad de carga del ambiente.

\section{Estacionalidad y ensamble de especies}

La variación estacional más marcada en densidad (Figura 2c) y abundancia de
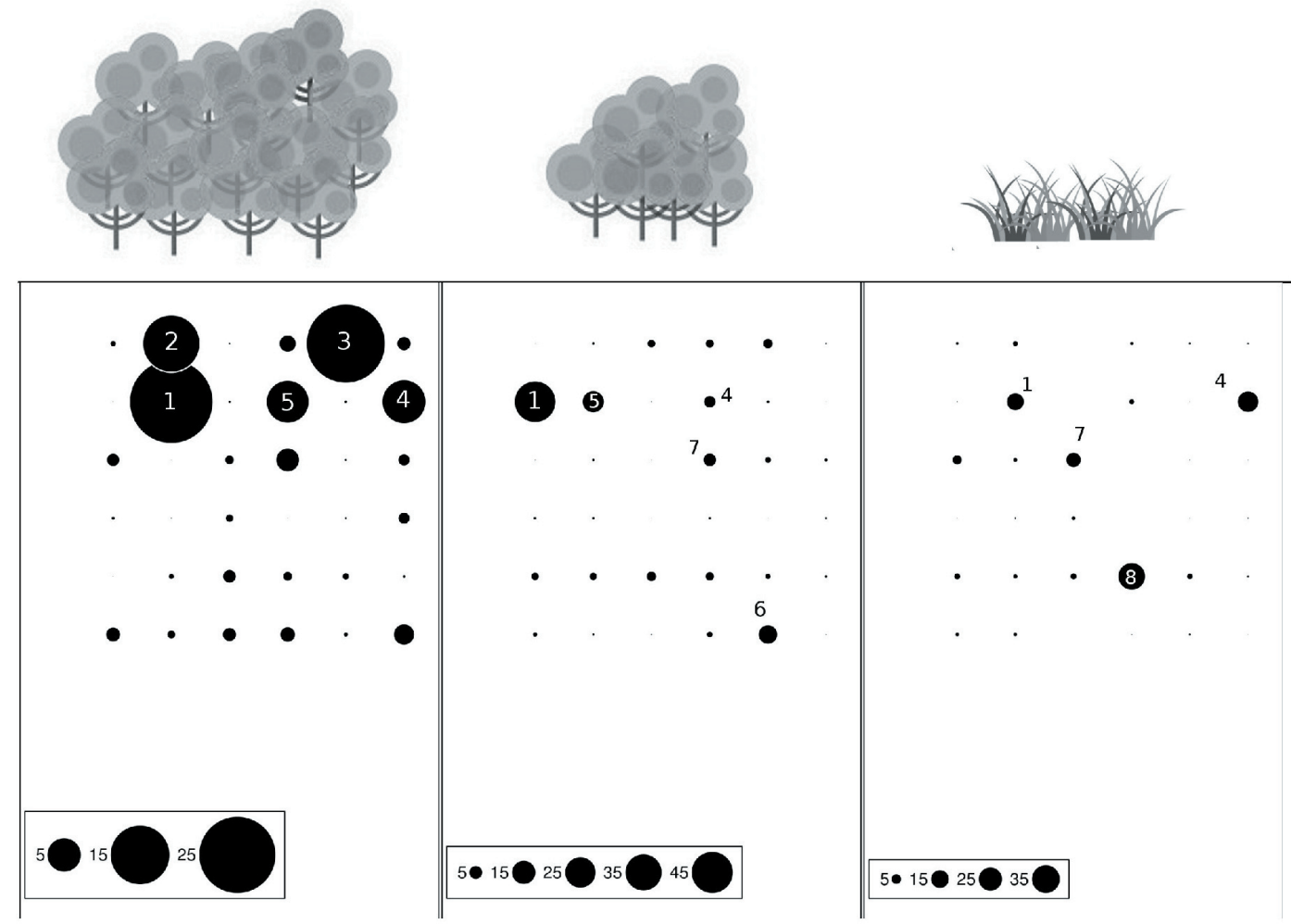

Figura 5. Diagrama comparativo de la distribución en abundancia de especies de oribátidos ponderado por la densidades media de individuos ( $\mathrm{Gr}=1 ; \mathrm{Ch}=0.5 ; \mathrm{Ma}=0.25$; ver resultados). $1=$ Pspi; $2=$ Sele; $3=$ Tmin; $4=\mathrm{Sacu} ; 5=\mathrm{Rmer}$; 6=Ecri; $7=$ Osur; $8=$ Hsur.

Figure 5. Comparative diagram of the oribatid mites abundance distribution weighed by the average density $(\mathrm{Gr}=1$; $\mathrm{Ch}=0.5 ; \mathrm{Ma}=0.25$; see results). $1=$ Pspi; $2=$ Sele; $3=$ Tmin; $4=\mathrm{Sacu} ; 5=$ Rmer; $6=$ Ecri; $7=$ Osur; $8=$ Hsur. 
oribátidos (Figura 4) se observó en la matriz. La vinculación estrecha entre los cambios de la comunidad de oribátidos y las características edáficas está muy bien sustentada (Block 1966; Al-Assiuty et al. 1993; Andres and AthiasBinche 1998; Hasegawa 2001), por lo que las alteraciones estacionales en la densidad de individuos de la matriz de pastizal se pueden vinculan a las variaciones anuales en temperatura, humedad y disponibilidad de materia orgánica en los suelos del pastizal. La ausencia de una marcada estacionalidad en los parches de tala está en relación con las condiciones más estables de este ambiente, derivadas en principio de la protección que ofrece la copa de los árboles al evitar la exposición directa de los rayos de luz y la consecuente pérdida de humedad del suelo.

La composición de especies de oribátidos del parche mayor fue temporalmente más estable a lo largo de las estaciones del año (Figura 3), con un elenco de 20 especies de estancia permanente (las denominadas ubicuas) vs. 10 en la matriz y el parche menor. Un punto a destacar es la gran cantidad de especies raras (12-16 especies) que en Gr representó el segundo grupo -y el primero en $\mathrm{Ch}$ y $\mathrm{Ma}$ - de importancia. Considerando la identidad de las especies y su ocurrencia a lo largo del año, se puede observar que algunas, como Multoribates aff. chavinensis y Quadroppia cristata se registraron como raras en los tres ambientes, lo que podría vincularse con ciertos requerimientos alimentarios $\mathrm{o}$, simplemente, con cuestiones de densidad poblacional. Otras especies (Nothrus anauniensis, $N$. beckiy Phthiracarus sp1) quese registraron como ubicuas o frecuentes en el parche mayor disminuyeron su abundancia y/o desaparecieron en la matriz. Badejo and Akinwole (2006) reportaron una situación similar para especies filogenéticamente relacionadas a las reportadas aquí: Nothrus lasebikaniy Mesoplophora ifeana. Los autores atribuyeron los cambios observados a las diferencias en el grado de exposición lumínica de los ambientes estudiados, lo que resulta congruente con las características de los ambientes estudiados aquí (i.e., diferencias en la humedad relativa; mayor incidencia lumínica de los suelos de la matriz). A nivel de composición de especies, un dato que aboga por la postura del ambiente como moderador de la comunidad de oribátidos está dado por la distribución de $P$. spinipes. Esta especie domina en ambos parches, pero en la matriz es desplazada por $H$. suramericanus, una especie con menos requerimientos específicos, distribución geográfica más amplia (Subías 2004) y que ha sido colectada en ambientes muy heterogéneos (Fredes and Martínez 2010).

En principio, los elementos hasta aquí discutidos dan la pauta de que la dinámica comunitaria de los oribátidos de Laguna de los Padres tendería a ser del tipo determinística, es decir, donde el establecimiento de las especies lo condicionan más las características ambientales que los eventos de migración y dispersión (Fukami 2010). Sin embargo, resta mucho para obtener una respuesta inequívoca acerca de las vinculaciones entre la estructura de la comunidad de oribátidos y las condiciones ambientales y habitacionales que permiten su establecimiento.

\section{CONCLUSIONES}

Las comunidades de oribátidos de Laguna de los Padres estudiadas estuvieron condicionadas principalmente por el tipo de ambiente (pastizal o bosque), hallándose comunidades más diversas y abundantes en los parches de bosques. En cuanto a su estructura, los ensambles de oribátidos presentaron patrones de distribución muy similares, pero con variaciones estacionales más marcadas en el pastizal.

En los talares, el área no influyó en el patrón de distribución de las especies, ni en la diversidad, ni la riqueza de especies. El tamaño del parche sí afectó la abundancia de las especies, determinando tamaños poblacionales menores en el parche menor. La evaluación de la fragilidad de estas poblaciones podría ser un punto de inicio a la hora de seleccionar áreas de conservación.

AgradecimiEnTos. La autora desea agradecer a los revisores y al editor de la revista por sus enriquecedores comentarios y sugerencias.

\section{REFERENCIAS}

Andres, P., and F. Athias-Binche. 1998. Decomposition of organic matter in two forest ecosystems (Natural Park of Montseny, Spain). I. Abundance, biomass and distribution of the soil microarthropods. Vie et Milieu 48:215-225.

Arturi, M., and J. Goya. 2004. Estructura, dinámica y manejo de los talares del NE de Buenos Aires. Pp. 1-23 en: M. Arturi, J. Frangi and J. Goya (eds.). Ecología y manejo de los bosques de Argentina. Editorial de la Universidad Nacional de La Plata, Buenos Aires.

Al-Assiuty, M. A. I., B. M. Bayoumi, M. A. Khalil, and N. M. Van Straalen. 1993. The influence of vegetational type on 
seasonal abundance and species composition of soil fauna at different localities in Egypt. Pedobiologia 37:210-222.

Badejo, M. A. 1990. Seasonal abundance of soil mites (Acarina) in two contrasting environments. Biotropica 22:382390

Badejo, M. A., and P. O. Akinwole. 2006. Microenvironmental preferences of oribatid mite species on the floor of a tropical rainforest. Exp Appl Acarol 40:145-156.

Balogh, J., and P. Balogh. 1988. Oribatid mites of the Neotropical Region I. Hungarian Natural History Museum, Amsterdam. Pp. 334.

Balogh, J., and P. Balogh. 1992. The oribatid mites genera of the world. Hungarian Natural History Museum, Berlin. Pp. 263.

Balogh, P., and J. Balogh. 1990. Oribatid Mites of the Neotropical Region II. Hungarian Natural History Museum, Berlin. Pp. 333.

Behan-Pelletier, V. M. 1999. Oribatid mite biodiversity in agroecosystems: role for bioindication. Agric Ecosyst Environ 74(1):411-423.

Block, W. 1966. Seasonal fluctuations and distribution of mite populations in moorland soils, with a note on biomass. J Anim Ecol 35:487-503.

Borcard, D. 1988. Les acariens oribates des sphaignes de quelques tourbières du Haut-Jura suisse. Thèse de doctorat. Faculté des Science. Université de Neuchêtel.

Cabrera, Á. L. 1971. Fitogeografía de la república Argentina. Soc Argent Bot Bol 14:1-42.

Coleman, D., D. Crossley, and P. Hendrix. 2004. Fundamentals of soil ecology. 2nd ed. Elsevier Academic Press. Pp. 386.

Debinski, D. M., and R. D. Holt. 2000. A survey and overview of habitat fragmentation experiments. Conserv Biol 14(2):342-355.

Ferreira, R. N. C., E. Franklin, J. L. Pereira De Souza, and J. De Moraes. 2012. Soil oribatid mite (Acari: Oribatida) diversity and composition in semi-deciduous forest fragments in eastern Amazonia and comparison with the surrounding savanna. J Nat Hist 46:2131-2144.

Fredes, N. A., and P. A. Martínez. 2010. Tamaño corporal y fecundidad de Hemileius suramericanus (Acari: Oribatida) en un bosque nativo del sudeste de Buenos Aires. Ecología Austral 20:293-301.

Fukami, T. 2010. Community assembly dynamics in space. Pp. 45-55 en: H. A. Verhoef and P. J. Morin (ed.). Community ecology. Proccess, Models and Applications. Oxford University Press. Pp. 247.

Gergócs, V., and L. Hufnagel. 2009. Application of oribatid mites as indicators. Appl Ecol Environ Res 7(1):79-98.

González, A., J. H. Lawton, F. S. Gilbert, T. M. Blackburn, and I. Evans-Freke. 1998. Metapopulation dynamics, abundance, and distribution in a microecosystem. Science 281(5385):2045-2047.

González, A., and E. J. Chaneton. 2002. Heterotroph species extinction, abundance and biomass dynamics in an experimentally fragmented microecosystem. J Anim Ecol 71(4):594-602.

Haene, E. 2006. Caracterización y conservación del talar bonaerense. Pp. 46-70 en: Mérida, E. and J. Athor (eds.). Talares bonaerenses y su conservación. Fundación de Historia Natural Félix de Azara, Buenos Aires.

Hasegawa, M. 2001. The relationship between the organic matter composition of a forest floor and the structure of a soil arthropod community. Euro J Soil Biol 37:281-284.

Hoyle, M., and A. R. Harborne. 2005. Mixed effects of habitat fragmentation on species richness and community structure in a microarthropod microecosystem. Ecol Entomol 30(6):684-691.

Hubert, J., A. Ku erová, and Z. M nzbergová. 2004. The comparison of oribatid mites (Acari: Oribatida) communities on various patches in the seminatural meadow. Ekológia (Bratislaba) 23(4):328-341.

Koleff, P., K. J. Gaston, and J. J. Lennon. 2003. Measuring beta diversity for presence-absence data. J Anim Ecol 72: 367-382.

Koteja, I., A. Stachowicz, and S. Sgardelis. 1994. Metapopulation study of Cryptostigmata inhabiting rock dwelling mosses. Bios (Macedonia Greece) 2:157-161.

Lussenhop, J. 1992. Mechanisms of microarthropod-microbial interactions in soil. Adv Ecol Research 23:1-33.

Magurran, A. E. 2004. Measuring Biological Diversity. Blackwell Publishing Ltd, Australia. Pp. 179.

Niedbała, W. 2004. Ptyctimous mites (Acari, Oribatida) of the Neotropical Region. Ann Zool 54:1-288.

Oksanen, J., F. Blanchet, R. Kindt, P. Legendre, P. R. Minchin, R. B. O'Hara, G. L. Simpson, P. Solymos, M. H. Stevens, and H. Wagner. 2011. Vegan: community ecology package. Pp. 190.

Parodi, L. R. 1940a. La distribución geográfica de los talares de la provincia de Buenos Aires. Darwiniana 4:33-56.

Parodi, L. R. 1940b. Los bosques naturales de la Provincia de Buenos Aires. A Acad Nac Cien Exac, Fís Nat 7:79-90.

Petersen, H., and M. Luxton. 1982. A comparative analysis of soil fauna populations and their role in decomposition processes. Oikos 39:288-388.

Saunders, D. A., Hobbs, R. J., and C. R. Margules. 1991. Biological consequences of ecosystem fragmentation: a review. Conserv Biol 5(1):18-32.

Stupino, S., M. Arturi, and J. Frangi. 2004. Estructura del paisaje y conservación de los bosques de Celtis tala Gill ex Planch del NE de la provincia de Buenos Aires. Rev Fac Agron, La Plata 105:37-45.

Subías, L.S., and P. Balogh. 1989. Identification keys to the genera of oppiidae Grandjean, 1951 (Acari: Oribatei). Acta Zool Hung 35:355-412. 
Subías, L. S. 2004. Listado sistemático, sinonímico y biogeográfico de los ácaros oribátidos (Acariformes, Oribatida) del mundo (1758-2002). Graellsia 60:3-305.

Torres Robles, S. S., and N. M. Tur. 2005. Los talares de la provincia de Buenos Aires. Pp. 246-250 en: A. Brown, U. Martinez Ortiz, M. Acerbi, and J. Corcuera (eds.). La situación ambiental Argentina 2005. Fundación Vida Silvestre Argentina, Buenos Aires.

Torres Robles, S. S., and M. F. Arturi. 2009. Variación de la composición y riqueza florística en los talares del Parque Costero del Sur y su relación con el resto de los talares bonaerenses. Pp. 104-121 en: Athor, J. (ed.). Parque Costero del Sur - Naturaleza, conservación y patrimonio cultural. Fundación de Historia Natural "Felix de Azara", Buenos Aires.

Torres Robles, S. S., and N. M. Tur. 2005. Los talares de la provincia de Buenos Aires. Pp. 246-250 en: La situación ambiental Argentina. Fundación Vida Silvestre Argentina, Buenos Aires.

Vervoorst, F. B. 1967. Las comunidades vegetales de la depresión del Salado (Provincia de Buenos Aires). Pp. 158-172 en: La vegetación de la República Argentina. Serie Fitogeográfica 7. Instituto nacional de Tecnología agropecuaria, Buenos Aires.

Swift, M. J., O. W. Heal, and J. M. Anderson. 1979. Decomposition in terrestrial ecosystems. University of California Press. Pp. 372.

Zaitsev, A. S., K. B. Gongalsky, T. Persson, and J. Bengtsson. 2014. Connectivity of litter islands remaining after a fire and unburnt forest determines the recovery of soil fauna. Appl Soil Ecol 83:101-108. 
Anexo. Lista de especies de oribátidos.

\section{Material Suplementario}

Annex. List of oribatid mites species.

\begin{tabular}{|c|c|c|}
\hline Familias & Acrónimo & Especie \\
\hline Achipteridae & Lqua & Lamellobates aff. quadricornis \\
\hline Anderemaeidae & Achi & Anderemaeus chilensis Hammer, 1962 \\
\hline Aphelacaridae & Asel & Adelphacarus sellnicki Grandjean, 1952 \\
\hline Austognetidae & Amul & Austrogneta multipilosa Balogh and Csiszár, 1963 \\
\hline Brachychthoniidae & Sels & Sellnickochthonius elsosneadensis (Hammer, 1958) \\
\hline Cosmochthoniidae & Tpul & Trichthonius pulcherrimus (Hammer, 1958) \\
\hline Dameoidae & Flac & Fosseremus laciniatus (Berlese, 1905) \\
\hline \multirow[t]{2}{*}{ Epilohmannidae } & Ecyl & Epilohmannia cylindrica (Berlese, 1904) \\
\hline & Epal & Epilohmannia pallida Wallwork, 1962 \\
\hline Eremulidae & Ecri & Eremulus crispus Hammer, 1958 \\
\hline Euphthiracaridae & Aard & Acrotritia ardua (Koch, 1841) \\
\hline \multirow{4}{*}{ Galumnidae } & Ccle & Carinogalumna clericata (Berlese, 1914) \\
\hline & Gcir & Galumna aff. circularis \\
\hline & Gpal & Galumna pallida Hammer, 1958 \\
\hline & Gret & Galumna reticulata Hammer, 1958 \\
\hline \multirow[t]{2}{*}{ Haplozetidae } & Pcap & Protoribates capucinus Berlese, 1908 \\
\hline & Rovu & Rostrozetes ovulum Berlese 1908 \\
\hline Machuellidae & Mven & Machuella ventrisetosa Hammer, 1961 \\
\hline Microzetidae & Bbra & Berlesezetes brazilozetoides Balogh and Mahunka, 1981 \\
\hline Mochlozetidae & Mros & Mancoribates rostropilosus Hammer, 1961 \\
\hline \multirow[t]{2}{*}{ Nothridae } & Nana & Nothrus anauniensis Canestrini and Fanzago, 1876 \\
\hline & Nbec & Nothrus becki Balogh and Mahunka, 1981 \\
\hline \multirow[t]{9}{*}{ Oppiidae } & Binc & Brachioppiella (Gressittoppia) incisa Martínez and Palacios-Vargas, 2006 \\
\hline & Galz & Graptoppia (Graptoppia) alzueti Martínez and Palacios-Vargas, 2006 \\
\hline & Kpil & Karenella pilisinopia Fredes and Martínez, 2014 \\
\hline & Lnod & Lanceoppia (Lancelalmoppia) nodosa (Hammer, 1958) \\
\hline & Mmin & Microppia minus (Paoli, 1908) \\
\hline & Onov & Oppiella nova (Oudemans, 1902) \\
\hline & Oxsp & Oxyoppia (Dzarogneta) sp. \\
\hline & Osur & Oxyoppia (Oxyoppiella) suramericana (Hammer, 1958) \\
\hline & Rmer & Ramusella (Inscultoppia) merimna (Balogh and Mahunka, 1977) \\
\hline Oribatellidae & Opro & Oribatella prolongata Hammer, 1961 \\
\hline Oribatulidae & Otra & Oribatula translineata (Mahunka, 1985) \\
\hline Oripodidae & Oaus & Oripoda australis Berlese, 1916 \\
\hline Otocepheidae & Ppau & Psedotocepheus pauliensis Pérez-Iñigo and Baggio, 1993 \\
\hline Phenopelopidae & Eacr & Eupelops acromios (Hermann, 1804) \\
\hline Phthiracaridae & Pht1 & Phthiracarus sp1 \\
\hline Protoribatidae & Tdis & Totobates discifer Hammer, 1961 \\
\hline Quadroppidae & Qcri & Quadroppia cristata Balogh and Mahunka, 1980 \\
\hline \multirow[t]{6}{*}{ Scheloribatidae } & Hsur & Hemileius suramericanus (Hammer, 1958) \\
\hline & Mcha & Multoribates cf chaviniensis \\
\hline & Sele & Scheloribates elegans Hammer, 1958 \\
\hline & Sacu & Scheloribates praeincisus acuticlava Pérez-Iñigo and Baggio, 1986 \\
\hline & Stal & Siculobata talarica Fredes and Martínez, 2013 \\
\hline & Upar & Urubambates paraguayensis Balogh and Mahunka, 1981 \\
\hline Suctobelbidae & Sden & Suctobelbila dentata (Hammer, 1961) \\
\hline \multirow[t]{2}{*}{ Tectocepheidae } & Tmin & Tectocepheus minor Berlese, 1903 \\
\hline & Tvel & Tectocepheus velatus (Michael, 1880) \\
\hline \multirow[t]{2}{*}{ Tegoribatidae } & Pspi & Physobates spinipes Hammer, 1958 \\
\hline & Tbor & Tectoribates borealis Behan-Pelletier and Walters, 2013 \\
\hline Teratoppidae & Tbre & Teratoppia brevipectinata Balogh and Mahunka, 1978 \\
\hline
\end{tabular}

